\title{
Erratum to: On the opportunity of using phylogenetic information to ask evolutionary questions in functional community ecology
}

\author{
Andreas Prinzing
}

Published online: 16 June 2016

(C) Institute of Botany, Academy of Sciences of the Czech Republic 2016

\section{Erratum to: Folia Geobot}

DOI 10.1007/s12224-016-9242-3

In the following paper: "On the opportunity of using phylogenetic information to ask evolutionary questions in functional community ecology" by Andreas Prinzing (DOI 10.1007/s12224-016-9242-3) the incorrect version of figure 1 was inadvertently used. Please find the correct figure $(1 \mathrm{a} / 1 \mathrm{~b})$ here.

Springer wishes to apologize for any inconvenience caused.

\footnotetext{
A. Prinzing $(\bowtie)$

University Rennes 1/Centre National de la Recherche

Scientifique, Research Unit "Ecosystèmes Biodiversité,

Evolution” («UMR 6553»), Campus Beaulieu, Bâtiment 14 A,

35042 Rennes, France

e-mail: Andreas.Prinzing@univ-rennes1.fr
} 
(a) Function of local communities in maintaining trait diversification Regional pool Local communties... ...maintaining trait evolution

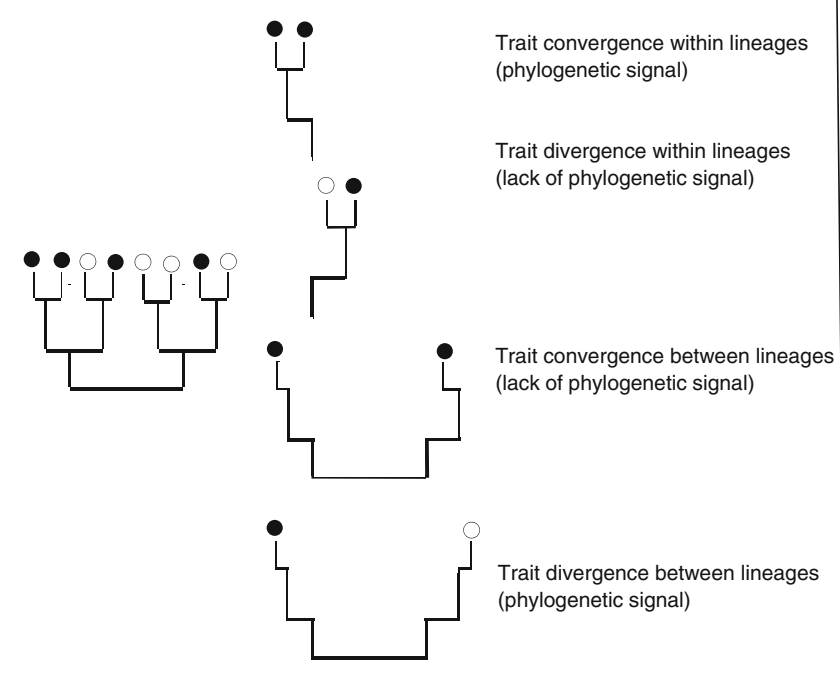

(b) Relating environments to trait diversification

Higher trait diversity

in a given environment.

.. may correspond to

different evolutionary scenarios

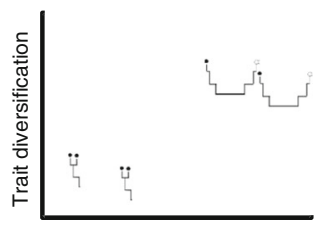

Environmental gradient

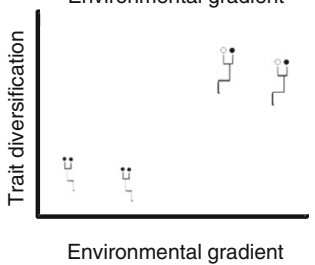

- 00

Environmental gradient

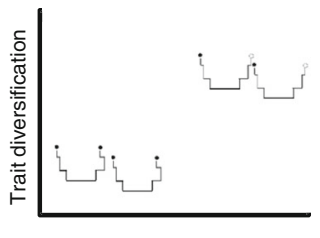

Environmental gradient

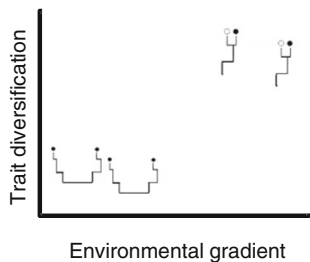

